\title{
Potential New Approaches for Diagnosis of Alzheimer's Disease and Related Dementias
}

\author{
R. Scott Turner ${ }^{1}$, Terry Stubbs ${ }^{2}$, Don A. Davies ${ }^{3 *}$ and Benedict C. Albensi ${ }^{3,4 *}$ \\ ${ }^{1}$ Department of Neurology, Georgetown University, Washington, DC, United States, ${ }^{2}$ ActivMed, Practices \& Research, \\ Methuen, MA, United States, ${ }^{3}$ Division of Neurodegenerative Disorders, St Boniface Hospital Research, University of \\ Manitoba, Winnipeg, MB, Canada, ${ }^{4}$ Department of Pharmacology \& Therapeutics, Max Rady College of Medicine, University \\ of Manitoba, Winnipeg, MB, Canada
}

Dementia is an umbrella term - caused by a large number of specific diagnoses, including several neurodegenerative disorders. Alzheimer's disease (AD) is now the most common cause of dementia in advanced countries, while dementia due to neurosyphilis was the leading cause a century ago. Many challenges remain for diagnosing dementia definitively. Some of these include variability of early symptoms and overlap with similar disorders, as well as the possibility of combined, or mixed, etiologies in some cases. Newer technologies, including the incorporation of PET neuroimaging and other biomarkers (genomics and proteomics), are being incorporated into revised diagnostic criteria. However, the application of novel diagnostic methods at clinical sites is plagued by many caveats including availability and access. This review surveys new diagnostic methods as well as remaining challenges - for clinical care and clinical research.

Keywords: memory, dementia, diagnostic, novel, clinic, Alzheimer

\section{INTRODUCTION}

Alzheimer's disease (AD), the most common cause of dementia, is now the 6th leading cause of death in the United States (Centers for Disease Control and Prevention). There are close to 50 million individuals with $\mathrm{AD}$ globally, and $\sim 6$ million individuals in the United States alone (Alzheimer's Disease International). Dementia is an umbrella term and may be caused by many disorders, including several neurodegenerative diseases. The differential diagnosis of dementia in older individuals typically includes $\mathrm{AD}$, vascular dementia $(\mathrm{VaD})$, dementia with Lewy bodies (DLB), Parkinson's disease dementia (PDD), frontotemporal dementia (FTD), and mixed dementias. Infectious etiologies of cognitive decline include neurosyphilis and HIV, and must be ruled out in the work-up of selected individuals. By definition, dementia requires a decline in more than one cognitive domain-memory, praxis, gnosis, language, visuospatial skills, executive function - and may also be accompanied by one or more behavioral disorders-depression, anxiety, personality changes, hallucinations, and delusions. The word dementia, taken from Latin, means "to take away one's mind," and is accompanied by significant social stigma in all cultures. The Diagnostic and Statistical Manual of Mental Disorders 5 (DSM-5, 2013) suggested deleting this demeaning term from our medical vocabulary, and proposed instead major neurocognitive disorder in order to minimize stigma and discrimination toward those affected (similar to the discontinuation of the demeaning term mongoloid in favor of Down syndrome). However, this attempt has mostly failed and most practitioners and advocacy groups still use the term dementia when referring to Alzheimer's disease and related conditions. 
Although the Greeks and Romans were well aware of dementia and associated it with aging, Alzheimer's disease (AD) was first reported in 1906 by the psychiatrist Alois Alzheimer. While at a Frankfurt hospital in Germany, Dr. Alzheimer examined his patient Auguste Deter, a 51 year-old woman, and described her as having progressive sleep and memory disturbances, confusion, paranoia, and aggression. Five years later upon her death Dr. Alzheimer, now in his Munich laboratory, investigated the patient's brain employing new silverstaining histological techniques to report the distinctive amyloid plaques and neurofibrillary tangles that continue to define $\mathrm{AD}$ pathologically. "A peculiar severe disease process of the cerebral cortex" was the title of Dr. Alzheimer's case report. The significance of these autopsy findings was not fully appreciated for many years, but the first diagnosis of AD was made. Now over a century later, brain autopsy remains the only way to diagnose definite $\mathrm{AD}$ (since brain biopsy is not a standard clinical practice).

Why do individuals with $\mathrm{AD}$ and similar progressive dementias often go undetected and undiagnosed? Diagnostic difficulties may be due in part to the variability of symptoms, some of which are difficult to identify. Also, dementia may be confused with other conditions including delirium, depression, anxiety, sleep disorders, side-effects of prescription and overthe-counter drug, drugs and alcohol abuse, and post-concussive syndrome. In addition, the onset of dementia is gradual and insidious, and patients may deny-or lack awareness of - their cognitive deficits. Moreover, given the global and regional variability in medical practice and cultural norms, consensus criteria for diagnosis remain controversial. Recent efforts in standardization of definitions and normal (vs. abnormal) values have resulted in greater harmonization of best practice. Controversies remain, and include the answer to questions such as: How much memory decline is acceptable and considered "normal aging"?

The diagnosis of a dementing illness is based on clinical signs and symptoms. After a medical history and physical examination, including a neurologic and psychiatric assessment, procedures employed to diagnose dementia may include neuropsychological testing, laboratory tests (blood and other biologic fluids), brain neuroimaging, and genetic testing. Methods for detecting changes in brain function and physiology are positron emission tomography (PET) and single photon emission computed tomography (SPECT) (1), which have been utilized in some clinical trials along with blood biomarkers. In some cases structural and functional magnetic resonance imaging (MRI), and to a lesser extent, computed tomography (CT) may be used as well. Typically, in the US and Canada, family medicine physicians, but more likely neurologists, geriatric psychiatrists, and geriatricians are trained in diagnosing individuals with dementia. A clinical diagnosis is important in order to determine prognosis, clinical management (including guiding appropriate prescription medications), genetic implications for family member, and clinical trial participation.

New recommendations to improve dementia detection and diagnosis were introduced by a committee of experts at the Alzheimer's Association's International Conference in Chicago, IL, USA, 2018. The steering committee known as
The Consortium for Detecting Cognitive Impairment, Including Dementia (DetectCID; https://www.detectcid.org/), was formed by the NIH with a goal to establish, test, and validate methods for detecting cognitive impairment in the public, including underrepresented populations. The purpose of this review is to survey novel methods and discuss potential challenges that clinicians face with regard to dementia diagnosis at clinical sites. Adoption of any novel methodology will be limited by practice standards, federal, state/provincial, and local government regulations, cost, and third-party coverage.

\section{BIOMARKERS}

Accumulating data has focused on discovering, evaluating, and validating biomarkers for application in clinical research. The goal in many cases is to provide evidence for earlier diagnostic and prognostic capability. Biomarkers are also employed to confirm and improve on diagnostic accuracy of dementia. In AD, biomarker development and validation has focused primarily on cerebrospinal fluid (CSF) -omics, including proteomics, and PET ligands to detect CNS amyloid beta $(\mathrm{A} \beta)$ or tau/tanglesthe two pathological hallmarks of AD (2-4). However, the use of CSF and PET biomarkers is limited by their invasiveness and cost, respectively. Other challenges concerning biomarker discovery and validation include collection methods, processing procedures, sample storage, and assay standardization within and across laboratories.

In response to these criticisms and concerns, an international working group (Alzheimer's Precision Medicine Initiative) was formed to review the current state-of-art for blood- based $\mathrm{AD}$ biomarkers (2). These would be preferable given that blood tests are more feasible in world-wide settings, are less costly (compared to PET) and less invasive (compared to lumbar puncture for CSF collection). To date, 19 bloodbased biomarkers were selected by the working group for additional consideration for $\mathrm{AD}$ detection. This working group also outlined a pathway from biomarker identification and development to validation so that academic-industrial partnerships in cooperation with regulatory bodies may codevelop putative blood-based AD biomarkers. Validation of biomarkers should start with assessment in a "black and white" panel study. Samples from patients with a diagnosis of $\mathrm{AD}$ would be compared to samples in neurologically healthy controls. This "black and white" study would aim to establish a concordance between the novel biomarker and the standard measure. This would be an attempt to validate the overall accuracy of the biomarker in a known group design. The next step is attempting to replicate the results in a set that more accurately reflects primary care. This second study would implement the technology from the developing laboratory and would involve technology transfer to an existing diagnostic assay that is widely available. The next step is refining the diagnostic algorithm, which would allow a case for potential regulatory approval. Finally, to establish interlaboratory replication, samples would be assayed on the intended equipment for regulatory approval. An optional step is the validation using CSF samples obtained 
from the same patients in the first study (2). Big data initiatives based on -omics data (Big Data Research and Development Initiative) analyzes large multidimensional blood- based omics data-allowing stratification of populations into well-defined subgroups (sharing commonalties) that may accelerate progress in biomarker development.

There are many challenges related to the validation of blood based biomarkers for dementia. Of 196 candidate blood based biomarkers, only 19 were prioritized for future consideration by the Alzheimer's Precision Medicine Initiative (2). However, none of the 19 blood based biomarkers were deemed to meet the target product profile. Most biomarker candidates were limited by lack of validation in external cohorts. The lack of external validation may lead to selective reporting and inflated predictive accuracy (5). Close cooperation is needed among academia, industry, and regulators to accelerate development of blood based biomarkers for clinical use. Biomarkers are often identified in academia and commercialization is executed by industry. Collaborations between academia and industry would allow for sharing of product testing, access to clinical data, and clinical endpoints (2).

\section{A/T/N System}

Some leading investigators propose that 7 biomarkers may be grouped into 3 categories based on their pathophysiology-the so-called A/T/N system (6) where "A" refers to $A \beta /$ amyloid based markers, " $\mathrm{T}$ " to tau/neurofibrillary pathology, and " $\mathrm{N}$ " to neurodegenerative or neuronal injury markers. This system uses the three categories and rates each category as either positive or negative. For example, a score could be $\mathrm{A}+/ \mathrm{T}+/ \mathrm{N}-$, which would indicate the person is positive for $A \beta$ and tau pathology, but negative for markers of neuronal injury or neurodegeneration.

The 7 biomarkers that are grouped into 3 binary categories include higher $\mathrm{A} \beta$ /amyloid deposits measured with PET tracer (7), and low CSF A $\beta$ (8-10). These biomarkers also include tau pathology with greater neurofibrillary tangles in CSF phosphorylated tau and a PET tracer of tau $(9,11)$. Finally, biomarkers of neurodegeneration or neural injury include total tau in CSF, hypometabolism measured with [18F]fluorodeoxyglucose ${ }^{18}$ (FDG)-PET, and atrophy on structural MRI in the hippocampus (12).

Biomarkers exist on a continuous scale from normal to abnormal demarcations to have diagnostic categorization of individuals informative for clinical decision making. These demarcations can be arbitrary and many individuals will have biomarkers close to the demarcations, which is true for most diseases and is not unique to $\mathrm{AD}$. The current biomarker measures are not sensitive to low but perhaps clinically significant levels of early pathology $(13,14)$. The \pm binary distinction is a convenient shorthand to increase communication that is easy to use and understand.

\section{NEUROIMAGING}

A variety of neuroimaging modalities have been developed with the goal of detecting dementia earlier along the $\mathrm{AD}$ spectrum and discriminating among the dementia differential diagnosis. The Alzheimer's Disease Neuroimaging Initiative (ADNI) (15) connects researchers across the US and Canada to collect, validate, and use pooled data (and samples), including MRI and PET neuroimaging, genetic data (genome-wide association study or GWAS), cognitive testing, CSF -omics, and blood-based biomarkers. Although the leading neuroimaging methods are PET and MRI, other modalities are employed such as computed tomography $(\mathrm{CT})(16,17)]$. Although CT is considered less sensitive than MRI for studies in dementia, CT is particularly useful for detecting bone lesions and new hemorrhage. Other advantages of CT over MRI include lower cost, shorter acquisition time, and no contraindication with claustrophobia or implanted metallic devices such as a pacemaker. SPECT, a nuclear imaging technique integrating $\mathrm{CT}$ and radioactive tracers, is also used in dementia diagnosis including, for example, differentiation of FTD from Jakob-Creutzfeldt Disease (JCD) (18). Functional MRI (fMRI) is a non-invasive technique that measures brain activity indirectly via changes in blood oxygenation. Functional MRI is useful in assessing integrity of brain networks in prodromal stages of $\mathrm{AD}$, thus detecting mild cognitive impairment (MCI) $(19,20)$ and in discriminating LBD from $\mathrm{AD}(21)$.

\section{TRANSCRANIAL MAGNETIC STIMULATION}

Transcranial magnetic stimulation (TMS) is a non-invasive therapeutic approach that uses a changing magnetic field to stimulate underlying nerve cells. TMS is under investigation for the treatment of a variety of neurological disorders, including dementia. Benussi et al. found that paired-pulse TMS distinguishes AD from FTD and healthy controls (HC) (22). In this study ( $n=175$ enrolled and underwent testing), TMS differentiated FTD $(n=64)$ from $\operatorname{AD}(n=79)$ with a sensitivity of $91.8 \%$ and specificity of $88.6 \%$. The authors propose that the observed difference was based on the activity of different intracortical circuits (i.e., cholinergic, GABAergic, and glutamatergic) in $\mathrm{AD}$ vs. FTD patients (both groups with mild disease). In other words, by using different TMS paradigms [short-latency afferent inhibition (SAI), short-interval intracortical inhibition (SICI), and intracortical facilitation (ICF)] one may assess the integrity of cholinergic, GABAergic, and/or glutamatergic cortical circuits. Overall, AD and FTD appeared to differ mainly in SICI-ICF and SAI activity where distinguishing $\mathrm{AD}$ and FTD from $\mathrm{HC}(n=32)$, resulted in a diagnostic accuracy of $>85 \%$.

TMS has also been used to discriminate between atypical Parkinsonian disorders (APD) and AD. In, Benussi et al. (23), APDs such as dementia with Lewy bodies (DLB; $n=27$ ), progressive supranuclear palsy (PSP; $n=13$ ) and corticobasal syndrome (CBS; $n=12$ ) were compared against $\mathrm{AD}(n=$ 63) and healthy controls (HC; $n=39)$. Similar to the TMS study discussed above, an $\mathrm{f}$ intracortical circuit activity using TMS paradigms was examined in these different groups. In this study, an overall diagnostic accuracy of $88.3 \%$ was found-with individual diagnostic accuracies as follows; $90.5 \%$ for $\mathrm{AD}, 85.2 \%$ for DLB, $76.0 \%$ for CBS-PSP, and $94.9 \%$ for HCs. Collectively 
these data suggest that TMS may be useful as a diagnostic tool to discriminate amongst various forms of dementia and other neurodegenerative disorders.

\section{ELECTROENCEPHALOGRAPHY}

Electroencephalographic (EEG) recordings have also been assessed for diagnosing dementia. EEG records electrical activity of cortical neurons and thus indirectly represents underlying brain function. EEG recording abnormalities are found in subcortical dementias, for instance, in DLB and PDD. Similar to other methods, the goal is to achieve earlier diagnosis with EEG, which is also a non-invasive technique. However, unlike PET or MRI scanning, EEG recordings are comparatively inexpensive and widely available at clinical centers. EEG methods are sometimes divided into two approaches. The first is accomplished in the resting state (awake at rest) in the absence of any stimulus. Since the patient is not required to perform a behavioral task, it is more comfortable and less stressful for patients (24). There are four effects of $\mathrm{AD}$ that have been reported in repeated studies in resting state EEG (25). There is a slowing of the power spectrum from high frequency (alpha, beta, gamma) to a low frequency in patients with $\mathrm{AD}$ (26). The shift from higher frequency to lower frequency is proportional to the progression of $\mathrm{AD}$. There is a reduction of EEG signal complexity in patients with $A D$, which is likely caused by neuronal death (27). Decreased in synchronization is observed in patients with $\mathrm{AD}$, which is a result of decreased connectivity between brain areas $(28,29)$. The cause of desynchronization is not well understood, it may emerge from atrophy of neural networks. There are neuromodulatory deficits in AD patients with their cross frequency interaction (30). For example, beta rhythms modulated at a theta rate is more pronounced in controls than in AD patients.

The second approach to EEG studies is conducted when the subject is performing a pre-defined task (task-oriented). This approach of task-oriented EEG studies is not ideal for most people with $\mathrm{AD}$ since patients have an increase of anxiety and anger. Therefore, performance of simple behavioral tasks may result in discomfort and inability to complete the task (31). In a study by Fraga et al. (32), EEG was used to discriminate among elderly healthy controls (HC; $n=27)$, MCI $(n=21)$ and $\mathrm{AD}(n$ $=15$ ). This study used EEG analysis during an executive function task (a working memory task). Significant differences were found and EEG was suggested to be useful for early MCI diagnosis, for improved $\mathrm{AD}$ diagnosis, and for assessing the probability of $\mathrm{MCI}$ progression to $\mathrm{AD}$.

The N100-P200 is elicited by presentation of a stimulus in the absence of task demands representing sensory processes as well as attention and peaks around $200 \mathrm{~ms}$ (33). While the traditional view was that the N100-P200 was mostly unaffected and therefore not a good biomarker of $\mathrm{AD}$, others have shown significantly longer latencies for the N100-P200 in familial AD (34). This indicates basic sensory and attentional processes may be compromised in AD.

Other forms of EEG have been tested for diagnosing dementia including quantitative electroencephalography (qEEG) - a computer-based method independent of traditional visual and subjective clinician's interpretation and based on statistical pattern recognition. Studies to date show a high diagnostic value of qEEG when evaluating subjects with $\mathrm{AD}, \mathrm{MCI}$, and other types of dementia. For example in a 2015 study by Engedal et al. (35), qEEGs distinguished $\mathrm{AD}$ patients from control subjects with a sensitivity of $84 \%$ and a specificity of $81 \%$. The qEEGs also separated patients with LBD or PDD from AD with a sensitivity of $85 \%$ and a specificity of $87 \%$. This study used a statistical pattern recognition method to analyze qEEG with a user-friendly score extracted from multiple qEEG features. The user-friendly features of this statistical pattern recognition would allow for translation into the clinical setting. The statistical pattern recognition method poorly separated patients with $\mathrm{AD}$ from those with MCI. However, in a more recent study by Hogh and colleagues (36), qEEG was used as a diagnostic tool in MCI $(n=56)$ and AD subjects $(n=32)$ vs. health controls (HC; $n=41$ ) across several sites in Denmark, Norway, and Sweden. Since the diagnostic and prognostic abilities in this study were low, it would not be appropriate for translation into a clinical setting. Overall however, the statistical pattern recognition method used in $\mathrm{qEEG}$ was superior to traditional EEG analysis. Also, the qEEG method correlated well with CSF AD biomarkers, suggesting an association with AD pathologies.

\section{ELECTROVESTIBULOGRAPHY}

Electrovestibulography (EVestG) is a vestibular-based diagnostic test that measures field potential activity recorded in the external ear canal in response to vestibular stimuli (37). The EVestG test is very similar to electrocochleography, but with the acoustic input replaced by a series of mechanically-driven orthogonal tilts accomplished by having the subject sit in a tilt chair (tilts in 2 dimensions-left/right and forward/backward). Recordings are made when the chair is static and also while moving. To date, EVestG methodology has been applied toward diagnosis and discrimination of PDD (Dastgheib et al. Med Biol Eng Comp, in press) vs. other neurological disorders, such as schizophrenia, depression, and Meniere's Disease (38-40). Overall, sensitivities and specificities have been typically above $85 \%$. EVestG is more than 95\% accurate in PDD diagnosis in patients that were at different stages of the disease (41). EVestG may provide a quick and non-invasive screening tool for PDD. Given the accuracy of PPD and PDD diagnosis, future research using EVestG should be conducted in other neurodegenerative disorders.

\section{CLINICAL DECISION SUPPORT}

Clinical support systems include computerized alerts, clinical guidelines, patient data reports, documentation templates, reference information, artificial intelligence (AI), automated historical comparisons, and diagnostic support tools (42-45). In particular, computer-based clinical decision support systems have evolved as a high tech tool for the objective evaluation and comparison of data for diagnostic purposes $(45,46)$. One 
example is the PredictND tool (47) that was recently tested in the Amsterdam Dementia Cohort $(n=504)$. In this 10 year study, PredictND was highly accurate in separating several types of dementias from each other (i.e., AD, FTD, DLB, or VaD) and from their respective controls (balanced accuracy 82.3\%). In addition to the predicted type of dementia, it also provided a confidence measure for classification. Accuracy was highest for $\mathrm{VaD}$ and lowest for DLB. In another recent study (48) across several sites in Europe, PredictND was used to differentiate among groups categorized as subjective cognitive decline [SCD; $n$ $=252)], \mathrm{AD}(n=138), \mathrm{DLB}(n=20), \mathrm{FTD}(n=34)$, and $\operatorname{VaD}(n$ $=23$ ). In this study, 747 patients completed follow-up visits. Of note, the etiological diagnosis changed in $13 \%$ of all cases when using PredictND, but the diagnostic accuracy did not change significantly. However, using the PredictND tool increased clinicians' confidence in their dementia diagnosis, indicating that computer-based support systems may assist with clinical decision making. PredictND uses data from neuropsychological tests, MRI, and CSF tests to classify patients according to the disease state index. Prospective studies have possible limitations. The study design was a tradeoff between retaining clinician's impression of patients and minimizing bias from the first to second session. The time between sessions was longer than intended, which may have affected the result. The follow up time was short, especially the evaluation progression of patients with MCI and SCD. However, this approach draws the clinician to data that are most relevant and removes the need to view tens or hundreds of data points individually.

In addition to the disease state index that PredictND used for diagnosis, others have used data from the ADNI to predict progression from MCI to $\mathrm{AD}$ (49). The patients underwent neuropsychological testing, MRI scanning, PET scanning, and CSF analysis. The ADNI analyzed MRI and PET scans in MCI patients using the multivariate technique of independent component analysis (ICA). ICA isolates unique features of biomarkers and potentially reveals patterns underlying the imaging data. ICA was able to predict the progression from MCI to AD (50). Support vector machine is a classification algorithm for pattern classification and predicted the progression of MCI to AD (51). However, even with the diagnostic accuracy mentioned in previous studies, clinical support systems are scarce. There is an absence of clear guidelines from regulatory bodies that impedes acceptance of clinical support systems (52). Developers of clinical support systems and its users should propose guidelines that will standardize clinical support systems. Input from both developers and users may result in more clinicians implementing clinical support systems.

Recently, AI applications have been growing. Deep learning is a type of AI that is sometimes described as simulating human learning approaches. Traditional PET image analysis requires an evaluation by experts trained in nuclear medicine and neuroimaging to make pattern recognition decisions. Therefore, deep learning algorithms theoretically may be used to learn and detect features or patterns in PET scans. In addition, deep learning may potentially help recognize additional patterns that are not as obvious during a human clinical review of scanned images. However, the usefulness of this task remains to be seen.
Also, given that traditional PET scan analyses are labor intensive, deep learning algorithms may shorten overall review time. In a recent ${ }^{18}$ FDG-PET study (53), it was hypothesized that a deep learning algorithm could detect patterns not evident on standard human-based clinical image review. PET images were obtained from the Alzheimer's Disease Neuroimaging Initiative (ADNI) database. The InceptionV3 architecture deep learning algorithm was trained on $90 \%$ of the ADNI data set and tested on the remaining $10 \%$, as well as the independent test set. The deep learning algorithm achieved a $82 \%$ specificity and $100 \%$ sensitivity. These findings imply that not only can deep learning algorithms predict the final diagnosis of AD with high accuracy and robustness, but they may also reduce overall cost due to shorter review times completed in part by machines. However, deep learning algorithms so far have been mainly utilized for the diagnosis of AD (54). Several technical challenges must be overcome to apply deep learning methods to other forms of dementia (54), neuroimaging datasets need a certain amount of labeling time to train a machine learning system, and various types of noise in the images reduces algorithm accuracy, to name a few. Some experts also state the best most AI systems do is reflect the past history's context for the current sample. Finally, once deep learning algorithms are optimized (85-95\%) to match specific types of human thinking, there may be no wiggle room left for "original" thought. These and other prognostic tools represent valuable support for clinicians. However, it is important to evaluate and compare performance in a standardized manner (55).

\section{OLFACTION AND TASTE}

Humans are capable of growing new nerve cells throughout life in a process called neurogenesis-suggesting a novel treatment strategy for dementia. To date, the two human brain regions that are sites of adult neurogenesis are a subfield of the hippocampus and the olfactory bulb (56). In fact, olfactory disorders may predict pre-dementia and dementia (57). Given this, it was hypothesized that olfaction and neurogenesis may be impaired in those with dementia, and that olfactory disorders may predict the conversion from MCI to AD dementia (57). Presently, no gold standard olfactory test is available for diagnosing or monitoring AD in clinical practice, but efforts have been made for predicting $\mathrm{AD}$ and for discriminating dementia diagnoses. For example, Williams et al. (58), found that that olfactory impairment was more pronounced in patients with mild DLB than in those with mild AD. Interestingly, in another study (59) patients with AD may demonstrate an asymmetrical decrement of odor detection sensitivity (left worse than right). In this study, the left-right nostril odor detection test functioned as an inexpensive, sensitive and specific test for probable AD.

The human sense of smell aligns with taste perception, and even vision to some degree (60). More recently (61, 62), taste cognition and taste detection were tested in subjects with suspected dementia. In one such study (62), the hypothesis was tested that the insula is associated with taste cognition in patients with $\mathrm{AD}(n=30)$ and $\operatorname{VaD}(n=20)$ vs. healthy controls $(n=$ 
15). Overall, it was concluded that glucose metabolism in the right insula was lower in the low taste cognition cohort and VaD patients with insular lesions showed impaired Taste Cognition Test results. Other recent studies (61) suggest that a failure of CNS taste processing occurs in patients with $\mathrm{AD}$.

\section{VISION}

Vision is impaired in dementia-with a variety of demonstrable impairment including contrast sensitivity (63). More recent studies $(64,65)$ examined whether a retinal examination may predict $\mathrm{AD}$ earlier and reveal disease progression $(66,67)$. Mahajan et al. (64), found ocular changes in $\mathrm{AD}$ besides decreased contrast sensitivity and included decreased vision, abnormal pupillary reaction, visual field changes, loss of retinal ganglion cells (and retinal nerve fiber layer), peripapillary atrophy, increased cup- disc ratio, retinal thinning, tortuosity of blood vessels, and the deposition of $\mathrm{A} \beta$ in the retina.

Examining color vision is also a potentially useful tool for discriminating different types of dementia. For instance, color vision discriminates $\mathrm{AD}$ from $\mathrm{DLB}$ (68). In this case, it was concluded that color vision deficits in patients with DLB showed a prevalence similar to the defining core features of DLB $(\sim 80 \%)$ and may be supportive of a diagnosis of DLB compared to AD. Other studies (69) found color vision differences when comparing $\mathrm{AD}$ to $\mathrm{VaD}$. In this study, the sensitivity/specificity analysis was $80.6 \%$ and $87.5 \%$ for discriminating $\mathrm{AD}$ vs. VaD.

Beta-amyloid deposits are found in the retina of patients with $\mathrm{AD}$ and are associated with a narrowed lumina and occlusion (70-73). Retinal photography was able to distinguish patients with $\mathrm{AD}$ and non-AD with $100 \%$ sensitivity and $84 \%$ specificity (74). The amyloid levels detected in the retina were correlated with amyloid levels in the brain via PET scan. An increase of $3.5 \%$ in retinal amyloid during a 3.5-months period suggests that retinal imaging could be used for monitoring the response to treatment (64). The retinal amyloid test is a screening tool that could complement currently used tests and potentially be used as part of regular eye exams.

\section{SALIVA}

Using saliva samples to diagnose $\mathrm{AD}$ has several advantages such as the non-invasive ease of acquisition and low cost. Chertkow et al. (75) used saliva and immunoblot analysis to quantify the phosphorylated tau (p-tau)/total tau (t-tau) ratio at different phosphorylation sites. Hyperphosphorylated tau (indicated by $\mathrm{p}$-tau) is a pathological marker for AD. In this study, samples were obtained from AD, MCI, and FTD patients. With one phosphorylation site, Ser-396, the p-tau/t-tau ratio was significantly increased in patients with $\mathrm{AD}$ compared with elderly control subjects. However, the sensitivity and specificity were not sufficiently robust to serve as a standard clinical biomarker. In fact, about one third of the $\mathrm{AD}$ group failed to show elevations of salivary tau. Another study with saliva (76) measured salivary acetylcholinesterase (AChE) activity in AD-an enzyme deficient in $\mathrm{AD}$ patients. The study examined in $15 \mathrm{AD}$ patients who were taking memantine vs. 15 healthy subjects. AChE activity in saliva in the AD group was indeed lower compared to the control group, but there was no significant difference between groups.

\section{SPEECH}

Speech impairment is well-known in $\mathrm{AD}$ (77) and other dementias and impairment in verbal communication depends on $\mathrm{AD}$ stage (78). Progression of speech impairments vary by individual, but three stages are identified (78). In the first, subjects demonstrate word-finding difficulties. In the intermediate stage, vocabulary and language become weaker. In the advanced stage, subjects provide only limited answers consisting of a few words. Nasrolahzadeh et al. (78), examined speech in AD subjects with the goal of utilizing spontaneous speech for earlier detection. This study focused on analyzing and comparing the quadratic phase coupling of spontaneous speech signals from healthy controls $(n=30)$ vs. AD subjects $(n=60)$ using bi-spectrum and bi-coherence methods. Signal processing methods of this type are statistical methods utilizing non-linear interactions of a continuous spectrum of propagating waves in one dimension. All participants were asked to tell "graceful personal stories, express their feelings, and converse in a friendly way." The results showed that the spontaneous speech signal of those with AD was significantly reduced compared to healthy controls.

In another study (79), speech samples were compared in probable $\mathrm{AD}$ subjects $(n=225)$ vs. probable $\mathrm{DLB}(n=67)$ subjects. In particular, speech samples were evaluated using the Cognitive Status Examination [COGNISTAT; formerly the Neurobehavioral Cognitive Status Examination (NCSE)], in which exam takers discuss what is happening between two people in a presented picture; however, other domains in addition to language may be tested, such as constructional ability, memory, calculation skills, and executive skills. During this test, subjects were scored (in a team effort by several psychologists) based on whether the subjects described or did not describe the relationship between two people during the speech sample. For instance, an example of the description group was as follows; "This is a picture of fishing. Someone is calling over. The person fishing does not notice a fish caught on the hook because he dozed off." In the no-description group, a typical answer may be " $A$ person is fishing. A person is performing acrobatics on the bridge." In addition, study participants were tested with the Mini-Mental State Examination (MMSE). The results suggest that patients with more severe overall cognitive dysfunction and also male patients are less likely to describe the relationship between two people. Difficulties with picture naming tasks are one of the most frequently reported speech impairments in people with $\mathrm{AD}(80)$.

Verbal fluency tests are one of the most widely used measures of speech function in patients with dementia (81). These tasks assess the person's ability to retrieve and produce words relevant for the specific task. Letter fluency records the generation of as many words as possible beginning with a given letter, for example words that begin with the letter S. Category fluency involves the generation of as many words as possible that fall into a specific category, for example tools. Letter and category fluency place 
demand on executive functioning since patients must engage in verbal retrieval and recall and inhibit incorrect responses. A meta-analysis of 153 studies with 15,990 AD patients found that $\mathrm{AD}$ patients had impaired letter fluency (82). Category fluency declines with the progression of $\mathrm{AD}$ (83).

Naming difficulty is another well-documented symptom of $\mathrm{AD}$ and it typically occurs early in disease onset (81). The Boston Naming Test (BNT) is a widely used test that comprises 60items ranging from frequent to infrequent items. The patient is presented with an item and allowed approximately $20 \mathrm{~s}$ to verbally identify the item. However, some patients with dementia find the 60 -item version difficult to complete due to their limited attention. Therefore, the BNT developed two 30-item versions that significantly correlated to each version and the 60 -item version. Differences have been found between patients with MCI and controls (84), and between AD and non-AD individuals (85).

A speech language pathologist can modestly improve communication for people with moderate to severe dementia (86). Speech language therapy may offer some protection against further speech decline. However, future studies should examine this question by measuring speech over a long period of time. Also, caregivers can be trained on the methods used by a speech language pathologist, which would lessen the time the patient must to be in the clinical setting.

\section{NEUROPSYCHOLOGIC TESTING}

Detailed comparator studies as well as comprehensive reviews addressing tests used for assessing cognitive status in $\mathrm{AD}$ and other dementias are published (87-97). Neuropsychologic tests may be organized by cognitive, functional, or behavioral domains (or their combinations) including activities of daily living (ADLQ), short mental status tests (MMSE, MoCA), brief dementia batteries (RBANS), behavioral symptoms (NPI-Q), clinical ratings (CDR), mood (Beck Depression Inventory II), IQ (Wechsler), executive function (Stroop test), visuoperceptual (drawing a clock), language or calculation (BDAE), and episodic memory (paragraph recall, word-list learning, ReyOsterreith Complex Figure). When administered as a battery, neuropsychological assessments quantify cognitive impairments and rates of progression. The CANTAB is a touchscreen computer automated neuropsychological test battery, which measures learning and memory. Patients with $\mathrm{AD}$ are impaired on the CANTAB test battery as compared to controls (98). Virtual reality is used to measure spatial navigation, which is impaired in people with dementia (99). The CANTAB and virtual reality could be integrated into family practice such that any medical professional could administer the task.

\section{CHALLENGES FOR IMPLEMENTATION AT CLINICAL RESEARCH SITES}

The goals of biomarker inclusion in newer diagnostic criteria include making a more accurate diagnosis of dementia. However, multiple barriers to implementation of innovative diagnostic methods and biomarkers limit their clinical application. These barriers include patient access to medical care, feasibility, cost, and third-party coverage.

Many individuals with dementia are never diagnosed by clinicians. Diagnostic nihilism stems in part from a widelyheld perception that currently available drug treatments for $\mathrm{AD}$ are inadequate - that they have minimal, if any, benefits, and that risk-benefit and cost-benefit analyses are negative. This current situation with dementia mimics a long-ago time when clinicians chose not to tell patients if they had terminal cancer. Clinicians should make an accurate dementia diagnosis, or refer to a specialty center as needed. If possible, biomarkers should be added to support a dementia diagnosis-as third-party coverage permits. An accurate diagnosis will determine prognosis, guide clinical care and management, enable a discussion of genetic risk with family members, and raise the possibility of clinical trial participation. Newer diagnostic technologies may be available if an individual screens or enrolls in a clinical study (for example, amyloid and tau PET scans, CSF proteomic analysis of $A \beta$ and tau, and ApoE genetic testing).

The development of newer biomarkers (for example, amyloid PET) has uncovered a 10-20 years prodrome of MCI and AD. This population - cognitively normal but at higher risk for $\mathrm{AD}$ is increasingly targeted for clinical research including prevention trials. The creation of databases composed of at-risk volunteers will aid recruitment for clinical studies. Recent efforts are also building trial-ready cohorts of well-characterized individuals in order to improve clinical trial efficiency and lower the high rates of screen-failure.

A significant challenge to implementation is the validation for use in a family practice setting. Clinical support systems need the guidelines of regulatory bodies and communication between the developers and clinicians to implement systems. There are many steps involved to validate blood based biomarkers including establishing a concordance between the novel biomarker and the standard measure, replication in an external laboratory, refinement, transfer to a commercial platform, and validation in an independent cohort. A similar process would be implemented to validate saliva based biomarkers. The computer based neuropsychological testing with an iPad touchscreen or virtual reality could be administered by any medical professional in the family practice setting.

\section{CONCLUSION}

In addition to a traditional medical history and neurologic examination, new technologies may assist in the diagnosis of dementia or impending dementia due to neurodegenerative disorders. Newer iterations of diagnostic criteria are incorporating validated diagnostic biomarkers, when available, as supportive evidence of a particular dementia diagnosis. Controversies remain, however, regarding the optimal biomarker, or combination of biomarkers, to include in these criteria. A lack of consensus of expert opinion, however, is not the only limitation to their clinical application. Operational issues 
including availability, feasibility, cost, and third-party coverage will limit their incorporation into clinical practice. Novel diagnostic biomarkers, particularly if relatively inexpensive and non-invasive, have the potential to markedly improve current practice, with added value in screening, prognosis, accurate diagnosis, and evaluation of novel treatments now under development for dementias including dementia due to $\mathrm{AD}$.

\section{AUTHOR CONTRIBUTIONS}

RT added a perspective from his memory clinic that he directs. TS added content relevant to CROs. BA who holds 2 dementia research chairs, was responsible for much of the research. DD revised a portion of the

\section{REFERENCES}

1. Pietrzak K, Czarnecka K, Mikiciuk-Olasik E, Szymanski P. New perspectives of alzheimer disease diagnosis - the most popular and future methods. Med Chem. (2018) 14:34-43. doi: 10.2174/1573406413666171002120847

2. Hampel H, O’Bryant SE, Molinuevo JL, Zetterberg H, Masters CL, Lista S, et al. Blood-based biomarkers for Alzheimer disease: mapping the road to the clinic. Nat Rev Neurol. (2018) 14:639-52. doi: 10.1038/s41582-018-0079-7

3. Chertkow H, Black S. Imaging biomarkers and their role in dementia clinical trials. Can J Neurol Sci. (2007) 34(Suppl. 1):S77-83. doi: 10.1017/s031716710000562x

4. Blennow $\mathrm{K}$, Zetterberg $\mathrm{H}$. Use of CSF biomarkers in Alzheimer's disease clinical trials. J Nutr Health Aging. (2009) 13:35861. doi: 10.1007/s12603-009-0043-8

5. Kerr NL. HARKing: hypothesizing after the results are known. Pers Soc Psychol Rev. (1998) 2:196-217. doi: 10.1207/s15327957pspr0203_4

6. Jack CR, Bennett DA, Blennow K, Carrillo MC, Feldman HH, Frisoni $\mathrm{GB}$, et al. $\mathrm{A} / \mathrm{T} / \mathrm{N}$ : an unbiased descriptive classification scheme for Alzheimer disease biomarkers. Neurology. (2016) 87:539-47. doi: 10.1212/WNL.0000000000002923

7. Klunk WE, Engler H, Nordberg A, Wang Y, Blomqvist G, Holt DP, et al. Imaging brain amyloid in Alzheimer's disease with pittsburgh compound-B. Ann Neurol. (2004) 55:306-19. doi: 10.1002/ana.20009

8. Fagan AM, Roe CM, Xiong C, Mintun MA, Morris JC, Holtzman DM. Cerebrospinal fluid tau/beta-amyloid(42) ratio as a prediction of cognitive decline in nondemented older adults. Arch Neurol. (2007) 64:3439. doi: 10.1001/archneur.64.3.noc60123

9. Mattsson $\mathrm{N}$, Zetterberg $\mathrm{H}$, Hansson $\mathrm{O}$, Andreasen $\mathrm{N}$, Parnetti L, Jonsson $\mathrm{M}$, et al. CSF biomarkers and incipient Alzheimer disease in patients with mild cognitive impairment. JAMA. (2009) 302:38593. doi: 10.1001/jama.2009.1064

10. Visser PJ, Verhey F, Knol DL, Scheltens P, Wahlund L-O, Freund-Levi Y, et al. Prevalence and prognostic value of CSF markers of Alzheimer's disease pathology in patients with subjective cognitive impairment or mild cognitive impairment in the DESCRIPA study: a prospective cohort study. Lancet Neurol. (2009) 8:619-27. doi: 10.1016/S1474-4422(09)70139-5

11. Buerger K, Ewers M, Pirttilä T, Zinkowski R, Alafuzoff I, Teipel SJ, et al. CSF phosphorylated tau protein correlates with neocortical neurofibrillary pathology in Alzheimer's disease. Brain. (2006) 129:303541. doi: 10.1093/brain/awl269

12. Besson FL, La Joie R, Doeuvre L, Gaubert M, Mézenge F, Egret S, et al. Cognitive and brain profiles associated with current neuroimaging biomarkers of preclinical Alzheimer's disease. J Neurosci. (2015) 35:1040211. doi: 10.1523/JNEUROSCI.0150-15.2015

13. Murray ME, Lowe VJ, Graff-Radford NR, Liesinger AM, Cannon A, Przybelski SA, et al. Clinicopathologic and 11C-Pittsburgh compound B implications of Thal amyloid phase across the Alzheimer's disease spectrum. Brain. (2015) 138:1370-81. doi: 10.1093/brain/awv050 manuscript. All authors contributed to the writing of the manuscript.

\section{FUNDING}

This research was funded by Canadian Institute of Health Research (CIHR), Grant No. PJT-162144, 514 and the St. Boniface Hospital Research Foundation, Grant Nos. 1406-3216 and $1410-3216$.

\section{ACKNOWLEDGMENTS}

We thank Mr. Gary Kibble for a critical reading of the manuscript.

14. Thal DR, Beach TG, Zanette M, Heurling K, Chakrabarty A, Ismail A, et al. [(18)F]flutemetamol amyloid positron emission tomography in preclinical and symptomatic Alzheimer's disease: specific detection of advanced phases of amyloid- $\beta$ pathology. Alzheimers Dement. (2015) 11:97585. doi: 10.1016/j.jalz.2015.05.018

15. Mueller SG, Weiner MW, Thal LJ, Petersen RC, Jack CR, Jagust W, et al. Ways toward an early diagnosis in Alzheimer's disease: the Alzheimer's Disease Neuroimaging Initiative (ADNI). Alzheimers Dement. (2005) 1:5566. doi: 10.1016/j.jalz.2005.06.003

16. Pasi M, Poggesi A, Pantoni L. The use of CT in dementia. Int Psychogeriatr. (2011) 23(Suppl. 2):S6-12. doi: 10.1017/S1041610211000950

17. Berti V, Pupi A, Mosconi L. PET/CT in diagnosis of dementia. Ann N Y Acad Sci. (2011) 1228:81-92. doi: 10.1111/j.1749-6632.2011.06015.x

18. Read SL, Miller BL, Mena I, Kim R, Itabashi H, Darby A. SPECT in dementia: clinical and pathological correlation. J Am Geriatr Soc. (1995) 43:12437. doi: 10.1111/j.1532-5415.1995.tb07400.x

19. Hojjati SH, Ebrahimzadeh A, Khazaee A, Babajani-Feremi A, Alzheimer's Disease Neuroimaging Initiative. Predicting conversion from MCI to AD by integrating rs-fMRI structural MRI. Comput Biol Med. (2018) 102:309. doi: 10.1016/j.compbiomed.2018.09.004

20. Machulda MM, Ward HA, Borowski B, Gunter JL, Cha RH, O'Brien PC, et al. Comparison of memory fMRI response among normal, MCI, and Alzheimer's patients. Neurology. (2003) 61:500-6. doi: 10.1212/01.wnl.0000079052.01016.78

21. Galvin JE, Price JL, Yan Z, Morris JC, Sheline YI. Resting bold fMRI differentiates dementia with Lewy bodies vs Alzheimer disease. Neurology. (2011) 76:1797-803. doi: 10.1212/WNL.0b013e31821ccc83

22. Benussi A, Di Lorenzo F, Dell'Era V, Cosseddu M, Alberici A, Caratozzolo S, et al. Transcranial magnetic stimulation distinguishes Alzheimer disease from frontotemporal dementia. Neurology. (2017) 89:665-72. doi: 10.1212/WNL.0000000000004232

23. Benussi A, Dell'Era V, Cantoni V, Ferrari C, Caratozzolo S, Rozzini L, et al. Discrimination of atypical parkinsonisms with transcranial magnetic stimulation. Brain Stimul. (2018) 11:366-73. doi: 10.1016/j.brs.2017. 11.013

24. Vecchio F, Babiloni C, Lizio R, Fallani FDV, Blinowska K, Verrienti G, et al. Resting state cortical EEG rhythms in Alzheimer's disease: toward EEG markers for clinical applications: a review. Suppl Clin Neurophysiol. (2013) 62:223-36. doi: 10.1016/b978-0-7020-5307-8.00015-6

25. Cassani R, Estarellas M, San-Martin R, Fraga FJ, Falk TH. Systematic review on resting-state EEG for alzheimer's disease diagnosis and progression assessment. Dis Markers. (2018) 2018:5174815. doi: 10.1155/2018/ 5174815

26. Jeong J. EEG dynamics in patients with Alzheimer's disease. Clin Neurophysiol. (2004) 115:1490-505. doi: 10.1016/j.clinph.2004.01.001

27. Jeong J, Kim SY, Han SH. Non-linear dynamical analysis of the EEG in Alzheimer's disease with optimal embedding dimension. Electroencephalogr Clin Neurophysiol. (1998) 106:220-8. doi: 10.1016/s0013-4694(97)00079-5 
28. Dunkin JJ, Leuchter AF, Newton TF, Cook IA. Reduced EEG coherence in dementia: state or trait marker? Biol Psychiatry. (1994) 35:8709. doi: 10.1016/0006-3223(94)90023-x

29. Wen D, Zhou Y, Li X. A critical review: coupling and synchronization analysis methods of EEG signal with mild cognitive impairment. Front Aging Neurosci. (2015) 7:54. doi: 10.3389/fnagi.2015.00054

30. Falk TH, Fraga FJ, Trambaiolli L, Anghinah R. EEG amplitude modulation analysis for semi-automated diagnosis of Alzheimer's disease. EURASIP J Adv Signal Proc. (2012) 2012:192. doi: 10.1186/1687-6180-2012-192

31. Kim J-S, Lee S-H, Park G, Kim S, Bae SM, Kim D-W, et al. Clinical implications of quantitative electroencephalography and current source density in patients with Alzheimer's disease. Brain Topogr. (2012) 25:46174. doi: 10.1007/s10548-012-0234-1

32. Fraga FJ, Mamani GQ, Johns E, Tavares G, Falk TH, Phillips NA. Early diagnosis of mild cognitive impairment and Alzheimer's with event-related potentials and event-related desynchronization in N-back working memory tasks. Comput Methods Programs Biomed. (2018) 164:113. doi: 10.1016/j.cmpb.2018.06.011

33. Katada E, Sato K, Ueda KOR. Cognitive event-related potentials: useful clinical information in Alzheimers disease. Curr Alzheimer Res. (2004) 1:63-9. doi: 10.2174/1567205043480609

34. Golob EJ, Ringman JM, Irimajiri R, Bright S, Schaffer B, Medina LD, et al. Cortical event-related potentials in preclinical familial Alzheimer disease. Neurology. (2009) 73:1649-55. doi: 10.1212/WNL.0b013e3181c1de77

35. Engedal K, Snaedal J, Hoegh P, Jelic V, Bo Andersen B, Naik M, et al. Quantitative EEG applying the statistical recognition pattern method: a useful tool in dementia diagnostic workup. Dement Geriatr Cogn Disord. (2015) 40:1-12. doi: 10.1159/000381016

36. Schjønning Nielsen M, Simonsen AH, Siersma V, Engedal K, Jelic V, Andersen $\mathrm{BB}$, et al. Quantitative electroencephalography analyzed by statistical pattern recognition as a diagnostic and prognostic tool in mild cognitive impairment: results from a nordic multicenter cohort study. Dement Geriatr Cogn Dis Extra. (2018) 8:426-38. doi: 10.1159/000490788

37. Lithgow B. A methodology for detecting field potentials from the external ear canal: NEER and EVestG. Ann Biomed Eng. (2012) 40:183550. doi: 10.1007/s10439-012-0526-3

38. Lithgow BJ, Garrett A, Heibert D. EVestG: a measure for Meniere's disease. Conf Proc IEEE Eng Med Biol Soc. (2008) 2008:4162-5. doi: 10.1109/IEMBS.2008.4650126

39. Haghgooie S, Lithgow BJ, Winograd-Gurvich C, Kulkarni J. EVestG: a diagnostic measure for schizophrenia. Conf Proc IEEE Eng Med Biol Soc. (2008) 2008:4142-5. doi: 10.1109/IEMBS.2008.4650121

40. Garrett A, Lithgow BJ, Gurvich C, Fitzgerald P. EVestG: responses in depressed patients. Conf Proc IEEE Eng Med Biol Soc. (2008) 2008:170710. doi: 10.1109/IEMBS.2008.4649505

41. Dastgheib ZA, Lithgow B, Moussavi Z. Diagnosis of Parkinson's disease using electrovestibulography. Med Biol Eng Comput. (2012) 50:48391. doi: 10.1007/s11517-012-0890-z

42. Stone EG. Unintended adverse consequences of a clinical decision support system: two cases. J Am Med Inform Assoc. (2018) 25:5647. doi: 10.1093/jamia/ocx096

43. Pflanzl-Knizacek L, Bergmoser K, Mattersdorfer K, Schilcher G, Baumgartner C. Development of a clinical decision support system in intensive care. Stud Health Technol Inform. (2018) 248:247-54.

44. Zikos D, DeLellis N. CDSS-RM: a clinical decision support system reference model. BMC Med Res Methodol. (2018) 18:137. doi: 10.1186/s12874-018-0587-6

45. Tomaszewski W. Computer-based medical decision support system based on guidelines, clinical pathways and decision nodes. Acta Bioeng Biomech. (2012) 14:107-16.

46. Heller I, Isakov A, Villa Y, Natour H, Inbar M, Fuchs J, et al. Evaluation of CaDet, a computer-based clinical decision support system for early cancer detection: a comparison with the performance of clinicians. Cancer Detect Prev. (2004) 28:352-6. doi: 10.1016/j.cdp.2004.06.004

47. Tolonen A, Rhodius-Meester HFM, Bruun M, Koikkalainen J, Barkhof F, Lemstra AW, et al. Data-driven differential diagnosis of dementia using multiclass disease state index classifier. Front Aging Neurosci. (2018) 10:111. doi: 10.3389/fnagi.2018.00111
48. Bruun M, Frederiksen KS, Rhodius-Meester HFM, Baroni M, Gjerum L, Koikkalainen J, et al. Impact of a clinical decision support tool on dementia diagnostics in memory clinics: the predictND validation study. Curr Alzheimer Res. (2019) 16:91-01. doi: 10.2174/1567205016666190103152425

49. Mattila J, Koikkalainen J, Virkki A, Simonsen A, van Gils M, Waldemar $\mathrm{G}$, et al. A disease state fingerprint for evaluation of Alzheimer's disease. $J$ Alzheimer's Dis. (2011) 27:163-76. doi: 10.3233/JAD-2011-110365

50. Shaffer JL, Petrella JR, Sheldon FC, Choudhury KR, Calhoun VD, Coleman $\mathrm{RE}$, et al. Predicting cognitive decline in subjects at risk for alzheimer disease by using combined cerebrospinal fluid, MR imaging, and PET biomarkers. Radiology. (2013) 266:583-91. doi: 10.1148/radiol.12120010

51. Cui Y, Liu B, Luo S, Zhen X, Fan M, Liu T, et al. Identification of conversion from mild cognitive impairment to alzheimer's disease using multivariate predictors. PLoS ONE. (2011) 6:e21896. doi: 10.1371/journal.pone.0021896

52. Belard A, Buchman T, Forsberg J, Potter BK, Dente CJ, Kirk A, et al. Precision diagnosis: a view of the clinical decision support systems (CDSS) landscape through the lens of critical care. J Clin Monit Comput. (2017) 31:261-71. doi: 10.1007/s10877-016-9849-1

53. Ding Y, Sohn JH, Kawczynski MG, Trivedi H, Harnish R, Jenkins NW, et al. A deep learning model to predict a diagnosis of alzheimer disease by using 18F-FDG PET of the brain. Radiology. (2019) 290:45664. doi: 10.1148/radiol.2018180958

54. Ahmed MR, Zhang Y, Feng Z, Lo B, Inan OT, Liao H. neuroimaging and machine learning for dementia diagnosis: recent advancements and future prospects. IEEE Rev Biomed Eng. (2019) 12:19-33. doi: 10.1109/RBME.2018.2886237

55. Bron EE, Smits M, van der Flier WM, Vrenken H, Barkhof F, Scheltens P, et al. Standardized evaluation of algorithms for computer-aided diagnosis of dementia based on structural MRI: the CADDementia challenge. Neuroimage. (2015) 111:562-79. doi: 10.1016/j.neuroimage.2015.01.048

56. Derby CD. Why have neurogenesis in adult olfactory systems? the presidential symposium at the 2006 achems conference. Chem Senses. (2007) 32:3613. doi: 10.1093/chemse/bjm011

57. Gros A, Manera V, De March CA, Guevara N, König A, Friedman $\mathrm{L}$, et al. Olfactory disturbances in ageing with and without dementia: towards new diagnostic tools. J Laryngol Otol. (2017) 131:572-9. doi: 10.1017/S0022215117000858

58. Williams SS, Williams J, Combrinck M, Christie S, Smith AD, McShane R. Olfactory impairment is more marked in patients with mild dementia with Lewy bodies than those with mild Alzheimer disease. I Neurol Neurosurg Psychiatry. (2009) 80:667-70. doi: 10.1136/jnnp.2008.155895

59. Stamps JJ, Bartoshuk LM, Heilman KM. A brief olfactory test for Alzheimer's disease. J Neurol Sci. (2013) 333:19-24. doi: 10.1016/j.jns.2013. 06.033

60. Bartoshuk LM. The functions of taste and olfaction. Ann N Y Acad Sci. (1989) 575:353-61. doi: 10.1111/j.1749-6632.1989.tb53256.x

61. Ogawa T, Irikawa N, Yanagisawa D, Shiino A, Tooyama I, Shimizu T. Taste detection and recognition thresholds in Japanese patients with Alzheimer-type dementia. Auris Nasus Larynx. (2017) 44:16873. doi: 10.1016/j.anl.2016.06.010

62. Suto T, Meguro K, Nakatsuka M, Kato Y, Tezuka K, Yamaguchi S, et al. Disorders of "taste cognition" are associated with insular involvement in patients with Alzheimer's disease and vascular dementia: "memory of food is impaired in dementia and responsible for poor diet." Int Psychogeriatr. (2014) 26:1127-38. doi: 10.1017/S1041610214000532

63. Bassi CJ, Solomon K, Young D. Vision in aging and dementia. Optom Vis Sci. (1993) 70:809-13. doi: 10.1097/00006324-199310000-00005

64. Mahajan D, Votruba M. Can the retina be used to diagnose and plot the progression of Alzheimer's disease? Acta Ophthalmol. (2017) 95:76877. doi: 10.1111/aos.13472

65. Cheung CY-L, Ikram MK, Chen C, Wong TY. Imaging retina to study dementia and stroke. Prog Retin Eye Res. (2017) 57:89-107. doi: 10.1016/j.preteyeres.2017.01.001

66. Guo J, Ni S, Li Q, Wang J-Z, Yang Y. Folate/Vitamin B alleviates hyperhomocysteinemia-induced alzheimer-like pathologies in rat retina. Neurosci Bull. (2019) 35:325-35. doi: 10.1007/s12264-018-0293-8

67. Tan Z, Ge J. Amyloid-beta, the retina, and mouse models of Alzheimer disease. Am J Pathol. (2010) 176:2055. doi: 10.2353/ajpath.2010.100076 
68. Flanigan PM, Khosravi MA, Leverenz JB, Tousi B. Color vision impairment differentiates alzheimer dementia from dementia with lewy bodies. J Geriatr Psychiatry Neurol. (2018) 31:97-102. doi: 10.1177/0891988718767579

69. Kawada T. Color vision test to differentiate Alzheimer's disease from vascular dementia. Int Psychogeriatr. (2017) 29:1753. doi: 10.1017/S10416102170 01089

70. Ellis RJ, Olichney JM, Thal LJ, Mirra SS, Morris JC, Beekly D, et al. Cerebral amyloid angiopathy in the brains of patients with Alzheimer's disease: the CERAD experience, Part XV. Neurology. (1996) 46:159296. doi: 10.1212/wnl.46.6.1592

71. Vinters HV, Wang ZZ, Secor DL. Brain parenchymal and microvascular amyloid in Alzheimer's disease. Brain Pathol. (1996) 6:179-95. doi: 10.1111/j.1750-3639.1996.tb00799.x

72. Jellinger KA. Alzheimer disease and cerebrovascular pathology: an update. $J$ Neural Transm (Vienna). (2002) 109:813-36. doi: 10.1007/s007020200068

73. Tian J, Shi J, Smallman R, Iwatsubo T, Mann DMA. Relationships in Alzheimer's disease between the extent of Abeta deposition in cerebral blood vessel walls, as cerebral amyloid angiopathy, and the amount of cerebrovascular smooth muscle cells and collagen. Neuropathol Appl Neurobiol. (2006) 32:332-40. doi: 10.1111/j.1365-2990.2006.00732.x

74. Frost S, Kanagasingam Y, Sohrabi H, Vignarajan J, Bourgeat P, Salvado O, et al. Retinal vascular biomarkers for early detection and monitoring of Alzheimer's disease. Transl Psychiatry. (2013) 3:e233. doi: 10.1038/tp.2012.150

75. Pekeles H, Qureshi HY, Paudel HK, Schipper HM, Gornistky M, Chertkow H. Development and validation of a salivary tau biomarker in Alzheimer's disease. Alzheimers Dement (Amst). (2019) 11:53-60. doi: 10.1016/j.dadm.2018.03.003

76. Bakhtiari S, Moghadam NB, Ehsani M, Mortazavi H, Sabour S, Bakhshi M. Can salivary acetylcholinesterase be a diagnostic biomarker for alzheimer? J Clin Diagn Res. (2017) 11:ZC58-60. doi: 10.7860/JCDR/2017/21715.9192

77. Lopez-de-Ipina K, Martinez-de-Lizarduy U, Calvo PM, Mekyska J, Beitia B, Barroso N, et al. Advances on automatic speech analysis for early detection of alzheimer disease: a non-linear multi-task approach. Curr Alzheimer Res. (2018) 15:139-48. doi: 10.2174/1567205014666171120143800

78. Nasrolahzadeh M, Mohammadpoory Z, Haddadnia J. A novel method for early diagnosis of Alzheimer's disease based on higher-order spectral estimation of spontaneous speech signals. Cogn Neurodyn. (2016) 10:495503. doi: 10.1007/s11571-016-9406-0

79. Matsunaga Y, Murayama N, Ota K, Fukase Y, Sato K, Iseki E, et al. Characteristics of dementia patients who described or did not describe the relationship between two people on the COGNISTAT speech sample. Appl Neuropsychol Adult. (2019) 26:482-7. doi: 10.1080/23279095.2018.1446015

80. Hodges JR, Salmon DP, Butters N. The nature of the naming deficit in Alzheimer's and Huntington's disease. Brain. (1991) 114 (Pt 4):154758. doi: 10.1093/brain/114.4.1547

81. Taler V, Phillips NA. Language performance in Alzheimer's disease and mild cognitive impairment: a comparative review. J Clin Exp Neuropsychol. (2008) 30:501-56. doi: 10.1080/13803390701550128

82. Henry JD, Crawford JR, Phillips LH. Verbal fluency performance in dementia of the Alzheimer's type: a meta-analysis. Neuropsychologia. (2004) 42:121222. doi: 10.1016/j.neuropsychologia.2004.02.001

83. Perry RJ, Watson P, Hodges JR. The nature and staging of attention dysfunction in early (minimal and mild) Alzheimer's disease: relationship to episodic and semantic memory impairment. Neuropsychologia. (2000) 38:252-71. doi: 10.1016/s0028-3932(99)00079-2

84. Dwolatzky T, Whitehead V, Doniger GM, Simon ES, Schweiger A, Jaffe D, et al. Validity of a novel computerized cognitive battery for mild cognitive impairment. BMC Geriatr. (2003) 3:4. doi: 10.1186/1471-2318-3-4

85. Blackwell AD, Sahakian BJ, Vesey R, Semple JM, Robbins TW, Hodges JR. Detecting dementia: novel neuropsychological markers of preclinical Alzheimer's disease. Dement Geriatr Cogn Disord. (2004) 17:42-8. doi: 10.1159/000074081

86. Swan K, Hopper M, Wenke R, Jackson C, Till T, Conway E. Speechlanguage pathologist interventions for communication in moderate-severe dementia: a systematic review. Am J Speech Lang Pathol. (2018) 27:83652. doi: 10.1044/2017_AJSLP-17-0043
87. Breton A, Casey D, Arnaoutoglou NA. Cognitive tests for the detection of mild cognitive impairment (MCI), the prodromal stage of dementia: metaanalysis of diagnostic accuracy studies. Int J Geriatr Psychiatry. (2019) 34:23342. doi: 10.1002/gps.5016

88. Aslam RW, Bates V, Dundar Y, Hounsome J, Richardson M, Krishan A, et al. A systematic review of the diagnostic accuracy of automated tests for cognitive impairment. Int J Geriatr Psychiatry. (2018) 33:561-75. doi: 10.1002/gps.4852

89. Chan JYC, Kwong JSW, Wong A, Kwok TCY, Tsoi KKF. Comparison of computerized and paper-and-pencil memory tests in detection of mild cognitive impairment and dementia: a systematic review and metaanalysis of diagnostic studies. J Am Med Dir Assoc. (2018) 19:74856. doi: 10.1016/j.jamda.2018.05.010

90. Creavin S, Wisniewski S, Noel-Storr A, Cullum S, MMSE review team. Cognitive tests to help diagnose dementia in symptomatic people in primary care and the community. Br J Gen Pract. (2018) 68:14950. doi: 10.3399/bjgp18X695249

91. Lee SH, Cho AJ, Min YK, Lee YK, Jung S. Comparison of the montreal cognitive assessment and the mini-mental state examination as screening tests in hemodialysis patients without symptoms. Ren Fail. (2018) 40:32330. doi: 10.1080/0886022X.2018.1455589

92. Tsatali M, Fotiadou F, Giaglis G, Tsolaki M. The repeatable battery for the assessment of the neuropsychological status (RBANS): a diagnostic validity study in Greek elderly. Aging Clin Exp Res. (2019) 31:130512. doi: 10.1007/s40520-018-1076-9

93. Holden HM, Milano NJ, Horner MD. Five-factor structure of the RBANS is supported in an Alzheimer's disease sample: implications for validation of neuropsychological assessment instruments. Appl Neuropsychol Adult. (2020) 27:232-42. doi: 10.1080/23279095.2018.1529671

94. Beath N, Asmal L, van den Heuvel L, Seedat S. Validation of the montreal cognitive assessment against the RBANS in a healthy South African cohort. $S$ Afr J Psychiatr. (2018) 24:1304. doi: 10.4102/sajpsychiatry.v24i0.1304

95. Allen CC, Ruff RM. Factorial validation of the Ruff-Light Trail Learning Test (RULIT). Assessment. (1999) 6:43-50. doi: 10.1177/107319119900600105

96. Federico A, Tinazzi M, Tamburin S. MoCA for cognitive screening in Parkinson's disease: Beware of floor effect. Mov Disord. (2018) 33:499. doi: $10.1002 / \mathrm{mds} .27329$

97. Kaur A, Edland SD, Peavy GM. The MoCA-memory index score: an efficient alternative to paragraph recall for the detection of amnestic mild cognitive impairment. Alzheimer Dis Assoc Disord. (2018) 32:1204. doi: 10.1097/WAD.0000000000000240

98. Egerházi A, Berecz R, Bartók E, Degrell I. Automated neuropsychological test battery (CANTAB) in mild cognitive impairment and in Alzheimer's disease. Prog Neuropsychopharmacol Biol Psychiatry. (2007) 31:746-51. doi: 10.1016/j.pnpbp.2007.01.011

99. Cogné M, Taillade M, N’Kaoua B, Tarruella A, Klinger E, Larrue F, et al. The contribution of virtual reality to the diagnosis of spatial navigation disorders and to the study of the role of navigational aids: a systematic literature review. Ann Phys Rehabil Med. (2017) 60:164-76. doi: 10.1016/j.rehab.2015.12.004

Conflict of Interest: RT: research support to Georgetown University from Lillly, Biogen, Roche, Genentech, Novartis, Janssen, and Eisai. TS: owner and CEO of ActivMed Practices and Research, Inc.; owner and CEO of ALLCUTIS Research, Inc.; owner and President of ActivMatters of the Brain, Inc. BA: medical advisor for Clinical Research IO. Medical advisor for Mitochondrial Transfusion.

The remaining author declares that the research was conducted in the absence of any commercial or financial relationships that could be construed as a potential conflict of interest.

Copyright (C) 2020 Turner, Stubbs, Davies and Albensi. This is an open-access article distributed under the terms of the Creative Commons Attribution License (CC BY). The use, distribution or reproduction in other forums is permitted, provided the original author(s) and the copyright owner(s) are credited and that the original publication in this journal is cited, in accordance with accepted academic practice. No use, distribution or reproduction is permitted which does not comply with these terms. 